\title{
Opportunities for serial femtosecond crystallography at SwissFEL
}

\author{
Karol Nass ${ }^{1}$ \\ ${ }^{1}$ Paul Scherrer Institut, Forschungsstrasse 111, Villigen, 5232, Switzerland \\ karol.nass@psi.ch
}

Serial femtosecond crystallography (SFX) developed at X-ray free-electron lasers (XFELs) is a successful method for structure determination of radiation sensitive microcrystals and for time-resolved crystallography (TR-SFX) ${ }^{1,2}$. A new XFEL facility (SwissFEL) was built and commissioned at the Paul Scherrer Institute (PSI) in Switzerland and started user operation in January 2019 ${ }^{3}$. To date, several user and commissioning SFX and TR-SFX experiments were successfully performed at SwissFEL. The overall beam stability, modest X-ray pulse parameters and excellent performance of the new JUNGFRAU X-ray detector developed at PSI contributed to their positive outcome. In this talk an overview of the facility with focus on the possibilities for SFX and TR-SFX experiments at SwissFEL will be presented. In particular, the supported sample delivery techniques (viscous and liquid jets, the SwissMX fixed target instrument), various sample environments, X-ray pulse properties, characteristics of the optical pump lasers and data analysis support will be discussed. Additionally, results from the first commissioning SFX experiment obtained with the new JUNGFRAU detector will be presented.

1. Schlichting, I. IUCrJ 2, 246-255 (2015).

2. Spence, J.C.H. IUCrJ 4, 322-339 (2017).

3. Milne, C.J. et al. App/ Sci-Basel 7, 720 (2017). 